\title{
Updates in palliative care - overview and recent advancements in the pharmacological management of cancer pain
}

\author{
Authors: Helen Wood, ${ }^{A}$ Andrew Dickman, ${ }^{B}$ Angela Star ${ }^{C}$ and Jason W Boland ${ }^{D}$
}

Pain is a common symptom in many types of cancer. Interdisciplinary team management, including pain assessment, explanation to the patient/family, treating the reversible, non-pharmacological treatments and reassessment are essential. This article focuses on the pharmacological management of cancer pain, and overviews and updates on the recent advances in this field. Both non-opioid and opioid analgesia as well as coanalgesics (adjuvants) are reviewed. Within non-opioid analgesia the risks of non-steroidal antiinflammatory drugs (NSAIDs) are considered and recommendations for NSAIDs in patients at risk of gastrointestinal and cardiovascular toxicity are made. For opioid analgesics, side effects of opioids are discussed alongside practical guidance on opioid prescribing and converting between opioids. Newer drugs such as tapentadol are considered in this update. Amitriptyline, duloxetine, gabapentin and pregabalin, and the guidance for their use are reviewed in the coanalgesics (adjuvants) section.

KEYWORDS: Pain, neoplasms, analgesics, opioid, tapentadol, nonsteroidal anti-inflammatory agents

\section{Background}

Patients receiving palliative care can have many different symptoms, including pain. An update on drugs for other symptoms is reviewed separately in this issue. ${ }^{1}$ Pain is a common symptom in many advanced illnesses. However, it is often poorly managed. ${ }^{2}$ Initial pain assessment (with the aim of finding a cause, assessing reversibility and the impact on the patient and their family, and to formulate a management plan), followed by reassessment of pain and effects / side effects of analgesics is vital. Explanation

Authors: ${ }^{\text {A }}$ specialty doctor in palliative care, Royal Liverpool and Broadgreen University Hospitals NHS Trust, Liverpool, UK; ${ }^{B}$ consultant palliative care pharmacist, Royal Liverpool and Broadgreen University Hospitals NHS Trust, Liverpool, UK; ' ${ }^{\text {specialist }}$ registrar in palliative medicine, Sheffield Teaching Hospitals, Sheffield, UK; ${ }^{\text {D }}$ senior clinical lecturer and honorary consultant in palliative medicine, Wolfson Palliative Care Research Centre, Hull York Medical School, University of Hull, Hull, UK to the patient/family, treating the reversible where appropriate and non-pharmacological treatments (often delivered by an interdisciplinary team) are essential, but outside the scope of the article. This review article will highlight recent updates in the pharmacological management of cancer pain in patients receiving palliative care.

Traditionally, the three-step World Health Organization (WHO) analgesic ladder has guided the pharmacological treatment of cancer pain. ${ }^{3}$ Although there is some evidence supporting the use of WHO recommendations in treating cancer pain, there is a lack of randomised controlled trial (RCT) data. ${ }^{4}$ Nonetheless, opioids such as codeine, morphine, oxycodone and fentanyl are often used in conjunction with paracetamol +/- NSAIDs and coanalgesics (adjuvants) to manage cancer pain. Other treatment modalities outside the WHO analgesic ladder (including interventional techniques like nerve blocks or intrathecal analgesia delivery) are also endorsed in the original WHO recommendations. ${ }^{4}$ In order to gain maximum benefit from these therapeutic options, they should be considered alongside the WHO analgesic ladder for patients likely to require them, rather than as a final step. ${ }^{4}$

\section{Non-opioid analgesics}

\section{Paracetamol}

A recent Cochrane review assessed the role of oral paracetamol in the management of cancer pain. ${ }^{5}$ There were three small RCT's included. All three studies assessed paracetamol in combination with opioids; there was high risk of bias. None assessed the use of paracetamol alone (first step). Overall, there was no high-quality evidence for or against using paracetamol (alone or with opioids) for cancer pain. ${ }^{5}$

\section{Non-steroidal anti-inflammatory drugs}

Non-steroidal anti-inflammatory drugs are used in palliative care for pain related to inflammation, which is an underlying aetiology in many people with cancer pain. ${ }^{2}$ The role of NSAIDs in cancer pain was assessed in a recent Cochrane review. ${ }^{6}$ Nine of the 11 included studies had substantial risk of bias, being small and having incomplete outcome data. In four studies, NSAIDS initially reduced moderate or severe pain after 1 or 2 weeks ( 415 participants). Overall, there was no high-quality evidence for or against the use of NSAIDs (alone or with opioids). 
Studies examining NSAID use in other conditions can provide baseline safety information, with the understanding that cancer and other serious illness carry additional NSAID toxicity risks. Non-steroidal anti-inflammatory drugs can have gastrointestinal (GI), renal and cardiovascular (CV) toxicity. ${ }^{2,7,8} \mathrm{~A}$ meta-analysis of RCTs, ${ }^{7}$ for all types of pain identified that COX-2 selective inhibitors (coxibs) increased major vascular events by a third compared to placebo (rate ratio $1.37,95 \%$ confidence interval [CI] 1.14-1.66, $p=0.0009$ ), mainly due to major coronary events. The vascular risk of different coxibs were similar. The risk of vascular death was significantly increased by coxibs $(1.58,95 \%$ CI 1.00-2.49, $p=0.0103)$ and diclofenac $(1.65,95 \%$ CI 0.95-2.85, $\mathrm{p}=0.0187$ ), increased by ibuprofen $(1.90,95 \%$ CI $0.56-6.41$, $\mathrm{p}=0.17)$, but not increased by naproxen $(1.08,95 \%$ CI $0.48-$ 2.47, $\mathrm{p}=0.80$ ).

The risk of upper GI complications, compared with placebo, was doubled with coxibs and diclofenac, and quadrupled with high doses of ibuprofen ( $2400 \mathrm{mg} / 24 \mathrm{hr}$ ) and naproxen (1000 mg/24 hr). Only $2 \%$ of upper GI complications were fatal. Heart failure risk leading to hospital admission was doubled by all NSAIDs. ${ }^{7}$ Celecoxib and diclofenac have a lower risk of upper GI complications but higher risk of major CV events. They should be avoided in patients with CV risk factors.

Patients with rheumatoid arthritis or osteoarthritis and increased CV risk were randomly assigned to receive celecoxib (100 mg twice a day), ibuprofen (600 mg three times a day) or naproxen (375 mg twice a day) with matching placebo. ${ }^{8}$ Cardiovascular death was not higher with moderate doses of celecoxib compared with nonselective NSAIDs (hazard ratio [HR] for celecoxib vs naproxen: 0.93, 95\% CI 0.76-1.130; HR for celecoxib vs ibuprofen: 0.85, 95\% CI 0.70-1.04, $p<0.001)^{8}$

Based on individual risk of GI or CV toxicity, the following recommendations are made about NSAID choice: ${ }^{7,9-11}$

> no CV or GI risk: non-selective NSAID (eg naproxen, ibuprofen, diclofenac)

> GI risk and no CV risk: avoid if possible; if essential celecoxib* (200 mg/24 h)

> CV risk +/- GI risk: avoid if possible; naproxen ( $1 \mathrm{~g} / 24 \mathrm{~h}$ ) or low dose ibuprofen (<1200 mg/24 h).

*Note: celecoxib use in patients with CV and GI risks is contraindicated by the manufacturer.

Proton pump inhibitors should be co-prescribed for all patients on regular NSAIDs/COX-2 inhibitors irrespective of GI risks. ${ }^{12}$ Only celecoxib has been shown to reduce mucosal harm throughout the entire GI tract. ${ }^{11}$ Celecoxib is most likely the optimum choice NSAID for patients receiving palliative care, as it has a lower GI risk, and a similar CV and renal impairment risk compared to nonselective NSAIDs. ${ }^{8}$

The lowest effective dose of all NSAID regimens should be used for the shortest duration. Treatment should be regularly reviewed and discontinued if no benefit or intolerable side effects develop. ${ }^{9}$ They should be used with caution in high-risk elderly patients because of GI, renal and CV toxic effects. ${ }^{13}$

Practice point: If an NSAID is needed in patients with CV risk factors, they should be prescribed naproxen ( $1 \mathrm{~g} / 24 \mathrm{~h}$ ) or low dose ibuprofen ( $<1200 \mathrm{mg} / 24 \mathrm{~h}$ ). In patients at very high risk of upper GI complications, NSAIDs should be avoided; for those in whom it is essential, celecoxib (200 mg/24 h) plus a proton pump inhibitor has been recommended.

\section{Opioid analgesics}

Weak opioids (eg codeine) have traditionally been prescribed before strong opioids (eg morphine) for the management for cancer pain. Codeine is, however, a prodrug of morphine, being metabolised by cytochrome P450 CYP2D6 to its active metabolite, morphine. Patients who have inactive copies of CYP2D6 (poor metabolisers) may find codeine is ineffective, while those with additional copies (ultrarapid metabolisers) are at risk of opioid toxicity. ${ }^{14}$ Starting a low-dose strong opioid (eg $\leq 30 \mathrm{mg} /$ day oral morphine) has been shown to give better pain relief than using weak opioids such as codeine. ${ }^{9,15}$ For patients being converted from a weak to a strong opioid, with normal renal and hepatic function, a typical starting dose is $10-15 \mathrm{mg}$ oral sustained-release morphine twice daily, plus as required $5 \mathrm{mg}$ oral immediaterelease morphine. ${ }^{9,16}$ For opioid naive patients, smaller doses of immediate-release morphine as required and/or modified-release morphine are generally prescribed, monitored and titrated to effect / side effects. ${ }^{9}$

\section{Choice of opioid}

European Association of Palliative Care guidance states there are no important differences between morphine, oxycodone and hydromorphone given by the oral route and suggests that any one of these three drugs can be used as the first-choice strong opioid for moderate to severe cancer pain. ${ }^{15}$ This was also illustrated in a Cochrane Review of 17 studies which suggested that oxycodone has similar analgesic effects and adverse events to other strong opioids and that oxycodone or morphine can be used as first-line oral opioids for relief of cancer pain. ${ }^{17}$ National Institute for Health and Care Excellence (NICE) guidance recommends morphine as first line in patients with advanced or progressive disease. ${ }^{16}$

> Practice point: NICE GC140 recommends that morphine should be used as first-line oral opioid for relief of cancer pain.

\section{Side effects}

Opioids have a range of potential side effects (Table 1). A recent overview of Cochrane Reviews evaluated adverse events after

Table 1. Side effects of therapeutic opioids. Reproduced with permission from Boland et a ${ }^{33}$

\section{System Effects}

Gastrointestinal Constipation, xerostomia, nausea and vomiting, gastro-oesophageal reflux

Neurological Delirium, hallucinations, sedation, myoclonus, hyperalgesia, seizures, headaches

Cardiovascular Bradycardia, hypotension

Pulmonary Respiratory depression, non-cardiogenic pulmonary oedema

Urological Urinary retention, altered renal function

Endocrinological Hypogonadism, sexual dysfunction, osteoporosis

Immune T cell, natural killer (NK) cell, neutrophil and monocyte dysfunction, cytokine dysregulation (clinical effect unknown) 
$\geq 2$ weeks opioid use for chronic non-cancer pain in adults. ${ }^{18}$ Fourteen reviews showed a significant increased risk of adverse events (including constipation, dizziness, drowsiness, fatigue, hot flushes, increased sweating, nausea, pruritus and vomiting) with opioids compared to both placebo (risk ratio [RR] 1.42, 95\% CI 1.22-1.66) and non-opioid analgesics (RR 1.21, 95\% CI 1.10-1.33). There was also a significant increased risk of experiencing a serious adverse event with opioids compared to placebo (RR 2.75, 95\% CI 2.06-3.67)

The various opioids have slightly different side effect profiles, partly due to their varying physicochemical and opioid receptor binding affinities. ${ }^{9}$ In chronic cancer pain, an RCT evaluating morphine, oxycodone, buprenorphine and fentanyl assessed adverse effects as a secondary outcome. ${ }^{19}$ Differences were observed for reactions involving the nervous system (confusion, hallucinations, myoclonus). Hallucinations occurred in $13.2 \%$ of patients taking morphine, but only $6.2 \%$ with oxycodone and buprenorphine, and $2.4 \%$ with fentanyl ( $p=0.001$ ). Severe myoclonus was not seen with oxycodone but occurred in $4.7 \%$ of cases with morphine $(p=0.029)$. High levels of confusion were less frequent with fentanyl (6.3\%) than with morphine (15.5\%), $(p=0.018)$. Drowsiness, constipation and dry mouth occurred in more than half the participants and equally among opioids. ${ }^{19}$ Other sources associate less constipation with the use of transdermal opioids. ${ }^{20-22}$

Some clinicians and patients are apprehensive about prescribing/ taking opioids as they are concerned that they might affect survival via immune and other mechanisms; this potentially leads to inadequate pain control. ${ }^{23-28}$ A systematic review reported that in the last days-to-weeks of life opioids did not influence survival, but there was a possible association between longer-term opioid use and shorter survival in adult patients with cancer. ${ }^{27}$ The studies that met the criteria for inclusion were mostly poor quality and the effect of opioids on survival was not the primary outcome, so causality cannot be established. ${ }^{27}$ Furthermore, the relationship between opioid use and shorter survival may be due to more aggressive disease being more painful, necessitating opioid use; the primary association might be between the more aggressive disease and shorter survival. ${ }^{29,30}$ Pain might also affect survival, so based on currently available data opioids should continue to be used. ${ }^{31,32}$

\section{Opioid prescribing considerations}

Morphine is generally avoided or used with extreme caution in patients with moderate to severe renal or hepatic disease, and specialist advice should be sought before prescribing strong opioids in these patients. ${ }^{16}$ In addition, the Faculty of Pain Medicine suggests that once a patient is on $120 \mathrm{mg} / \mathrm{day}$ oral morphine or equivalent, specialist advice should be sought as the risk of harm substantially increases beyond this dose. ${ }^{34}$ Side effects such as nausea, constipation and drowsiness and signs of toxicity should be discussed with patients when starting any opioid and patient preference considered. ${ }^{16}$

Practice point: Once a patient is on $120 \mathrm{mg} /$ day oral morphine or equivalent, specialist advice should be sought. Seek specialist advice in patients with renal or hepatic disease.

\section{Transdermal opioids}

Buprenorphine and fentanyl are very potent strong opioids, often used as transdermal patches (Table 2). They can be used
Table 2. Opioid equianalgesic ratios ${ }^{\circ}$

\begin{tabular}{|c|c|c|}
\hline $\begin{array}{l}\text { Conversion } \\
\text { (from PO } \\
\text { morphine) }\end{array}$ & $\begin{array}{l}\text { Potency } \\
\text { (compared with } \\
\text { PO morphine) }\end{array}$ & Example \\
\hline Codeine PO & 0.1 & $\begin{array}{l}\text { Codeine } 240 \mathrm{mg} \mathrm{PO} \\
=\text { morphine } 24 \mathrm{mg} \mathrm{PO}\end{array}$ \\
\hline Tramadol PO & $0.1-0.2$ & $\begin{array}{l}\text { Tramadol } 400 \mathrm{mg} / 24 \mathrm{~h} \text { PO } \\
=\text { morphine } 40-80 \mathrm{mg} / 24 \\
\text { h PO }\end{array}$ \\
\hline $\begin{array}{l}\text { Oxycodone } \\
\text { PO }\end{array}$ & $1.5-2$ & $\begin{array}{l}\text { Morphine } 60 \mathrm{mg} / 24 \mathrm{~h} \mathrm{PO} \\
=\text { oxycodone } 30-40 \mathrm{mg} / 24 \\
\text { h PO }\end{array}$ \\
\hline $\begin{array}{l}\text { Buprenorphine } \\
\text { TD }\end{array}$ & $70-115$ & $\begin{array}{l}\text { Morphine } 40-60 \mathrm{mg} / 24 \mathrm{~h} \text { PO } \\
=\text { buprenorphine } 600 \mathrm{mcg} / 24 \\
\mathrm{~h}=25 \mathrm{mcg} / \mathrm{h} \text { patch }\end{array}$ \\
\hline Fentanyl TD & 100-150 & $\begin{array}{l}\text { Morphine } 60-90 \mathrm{mg} / 24 \mathrm{~h} \text { PO } \\
=\text { fentanyl } 600 \\
\mathrm{mcg} / 24 \mathrm{~h}=25 \mathrm{mcg} / \mathrm{h} \text { patch }\end{array}$ \\
\hline Morphine SC & 2 & $\begin{array}{l}\text { Morphine } 60 \mathrm{mg} / 24 \mathrm{~h} \text { PO } \\
=\text { morphine } 30 \mathrm{mg} / 24 \mathrm{~h} \mathrm{SC}\end{array}$ \\
\hline \multicolumn{3}{|c|}{$\begin{array}{l}\text { aModified with permission from the Palliative Care Formulary 6th edition, } \\
\text { palliativedrugs.com Ltd, Nottingham, UK. Some conversions are not agreed } \\
\text { upon and expert opinion/local practice thus varies. PO = oral; TD = transdermal; } \\
\text { SC = subcutaneous }\end{array}$} \\
\hline
\end{tabular}

when oral opioids are not tolerated, when patients are unable to swallow or when there are compliance issues. In most cases patients also take an oral immediate release preparation of morphine or oxycodone as a rescue dose for breakthrough pain. ${ }^{20}$ National Institute for Health and Care Excellence guidelines do not recommend transdermal opioids as a first-line treatment when oral opioids are appropriate. ${ }^{16}$ Inability to rapidly titrate dosages makes these patches unsuitable for use in severe uncontrolled pain. ${ }^{16}$

Effective systemic analgesic concentrations of transdermal fentanyl are generally reached within 3 to 23 hours, with steady state plasma concentrations achieved within 36 to 48 hours. ${ }^{9}$ After 48 hours, if patients are requiring two or more rescue doses/day to effectively treat breakthrough pain, they should be assessed for a dose increase (by $12-25 \mathrm{mcg} / \mathrm{h}$ ). ${ }^{9}$

Effective systemic analgesic concentrations of transdermal buprenorphine are generally reached within 11 to 24 hours, with steady state being reached after 1 to 3 days (depending on preparation). ${ }^{9}$ After 72 hours, if patients are requiring two or more rescue doses/day to effectively treat breakthrough pain, they should be assessed for a dose increase to the next available strength. ${ }^{9}$

Buprenorphine and fentanyl are both associated with less constipation than equianalgesic doses of slow-release morphine and buprenorphine has been shown to cause less cognitive dysfunction than other opioids. ${ }^{20-22}$

Practice point: Buprenorphine and fentanyl are both associated with less constipation than equianalgesic doses of slow-release morphine. The lowest patch strength of buprenorphine ( $5 \mathrm{mcg} / \mathrm{h}$ ) is suitable for opioid naive patients; the lowest patch strength of fentanyl $(12 \mathrm{mcg} / \mathrm{h})$ is not suitable for opioid-naive patients. 


\section{Opioid-induced hyperalgesia}

Patients can paradoxically show an increased sensitivity to painful stimuli with increasing doses of opioids. This is termed opioid-induced hyperalgesia (OIH). Pain may be experienced in a different location and be of a different quality than the original pain. It generally occurs with high or rapidly increasing doses. ${ }^{35}$ If $\mathrm{OIH}$ is suspected, a specialist should be contacted for advice. Management of $\mathrm{OIH}$ can involve changing the opioid, a reduction in the dose (by 25 to $50 \%$ ) and addition of non-opioid analgesics. $^{9,35}$

\section{Opioid switching}

There is varying efficacy and tolerability to different opioids. Thirty percent of patients on morphine fail to achieve satisfactory analgesia (despite escalating doses) or experience intolerable side effects. ${ }^{36}$ Opioid switching has been shown to be effective in more than $80 \%$ of cancer patients with a poor response to an initial opioid. ${ }^{37}$ Patients who are stable on an opioid for a significant period of time may require increasing doses due to disease progression and/or tolerance. Drug tolerance may develop to both the analgesic and the central effects (eg drowsiness) of the drug, while tolerance to the peripheral side effects of the drug (eg constipation) is less common. Incomplete 'cross-tolerance' may allow the use of lower doses of alternative opioids permitting adequate analgesia without significant side effects. ${ }^{38}$ Switching is not only used for OIH but also intolerable side effects, patients who develop renal failure and patients with poor adherence to certain opioids.

An RCT compared the clinical analgesic response to oral morphine vs oral oxycodone as first-line treatment for cancerrelated pain. ${ }^{36}$ In the study, non-responders to the first opioid were switched to the alternative opioid, and their analgesic response was also evaluated. Sixty-two percent of patients randomised to morphine as the initial opioid had a good clinical response vs $67 \%$ who were randomised to oxycodone. There was no significant difference in clinical response rates to first-line opioid $(p=0.48)$. Patients who did not respond to the first-line opioid were switched. Fifty-two percent of patients switched from morphine to oxycodone responded. Sixty-seven percent of patients switched from oxycodone to morphine responded. There was no difference in overall response rates depending on which drug was used first $(p=0.81)$. This study shows that opioid switching can be used effectively in patients who do not respond to a first-line opioid.

When switching between different opioids, the dose of the new opioid should be reduced by about $25 \%$ (consult local guidance and/or specialist advice) to account for incomplete cross-tolerance. ${ }^{35}$

\section{Using equianalgesic tables}

Opioid conversion ratios are a guide only. See Table 2 for some common conversion ratios; more detailed tables can be found elsewhere (eg PCF $6{ }^{9}$ and the Palliative Care Adult Network guidelines ${ }^{39}$ ). Consult local guidance and/or specialist advice when changing patients' opioids.

Equianalgesic doses may differ due to interpatient variability secondary to drug pharmacokinetics, drug interactions, genetic differences and comorbidities, as well as drug manufacturer. ${ }^{9}$ Careful monitoring is needed during conversion to prevent under dosing or excessive dosing.

\section{Tapentadol}

A recent alternative to traditional strong opioids is tapentadol; licensed for both moderate to severe acute pain requiring opioids and severe chronic pain including cancer pain. Tapentadol is a weak $\mu$-opioid receptor agonist (18 times less potent binding affinity than morphine) and a noradrenaline reuptake inhibitor. ${ }^{40}$ The two distinct pharmacological actions are thought to synergistically enhance the descending inhibitory pain pathway. ${ }^{41}$

A Cochrane review of oral tapentadol in patients with cancer pain, included four studies (<200 patients per treatment arm) comparing prolonged-release tapentadol with modified-release morphine or oxycodone; meta-analysis was not possible. ${ }^{42}$ The review concluded that tapentadol was neither superior nor inferior to oxycodone or morphine.

Although tapentadol is a weak opioid, its side-effect profile is similar to strong opioids. Recent reviews report decreased GI-related side effects (nausea, vomiting and constipation) with tapentadol used for both cancer and chronic pain, when compared to strong opioids. ${ }^{40,43,44}$ Dry mouth and headache were reported more often than with oxycodone or placebo. ${ }^{44}$

> Practice point: Tapentadol prolonged-release should be considered an alternative for patients in whom traditional strong opioids have failed to provide adequate pain control, especially when doses are limited by intolerable side effects.

\section{Coanalgesics}

Coanalgesic (or adjuvant) medications refer to drugs which were originally marketed for indications other than pain. These include antidepressants such as amitriptyline and duloxetine, and anti-epileptics such as gabapentin and pregabalin. The number of these drugs has increased substantially over the last decade. Duloxetine is a serotonin-noradrenaline reuptake inhibitor that is licensed for treatment of diabetic peripheral neuropathic pain. In a multicentre randomised crossover trial of duloxetine vs placebo, ${ }^{45}$ it significantly reduced the average pain score in patients with painful chemotherapy-induced peripheral neuropathy from platinum and taxane agents. The duloxetine-first arm reported a mean decrease in average pain of 1.06 (95\% CI $0.72-1.40)$ vs 0.34 (95\% CI $0.01-$ $0.66)$ among those who received placebo $(p=0.003)$. The observed mean difference in average pain score between the duloxetine-first and placebo-first groups was 0.73 (95\% CI 0.26-1.20). Duloxetine also improved quality of life scores and decreased numbness and tingling. Although an intention-to-treat analysis was done, the dropout rate due to adverse events in the duloxetine first group was $11 \%$ vs $1 \%$ in the placebo group $(p<0.001) .45$

National Institute for Health and Care Excellence guidance recognises that many of the treatments used to treat neuropathic pain are unlicensed and this limits their use outside of specialist settings. ${ }^{46}$ The guidance suggests using one of amitriptyline, duloxetine, gabapentin or pregabalin as initial treatment for neuropathic pain (except trigeminal neuralgia). If initial treatment is not effective or not tolerated, it is advisable to change to a different class of drug and change again if second and third drugs are not effective. Combination therapy may also be a helpful option if the initial choices do not sufficiently improve pain. ${ }^{46}$

A double-blind RCT assessed the clinical efficacy of pregabalin, amitriptyline and gabapentin in neuropathic cancer pain compared to placebo. ${ }^{47}$ All three drugs were effective in relieving cancer- 
related neuropathic pain, with statistically and clinically significant morphine sparing effect of pregabalin in relieving neuropathic cancer pain and neuropathic symptoms compared to the other antineuropathic medications. ${ }^{47}$ However, a systematic review (which included the above study as the only RCT) appraised the literature of pregabalin in the treatment of neuropathic pain resulting from cancer or cancer treatment and found there were limited published data reporting efficacy and safety outcomes for pregabalin in the treatment of neuropathic pain in adults with cancer. ${ }^{48}$ Due to limitations within the studies included in the review, the authors felt it was not possible to draw any conclusions on pregabalin for the treatment of cancer-related neuropathic pain.

A meta-analysis of four RCTs examined whether combining opioids with pregabalin or gabapentin, compared to opioid monotherapy improved cancer pain. ${ }^{49}$ There was no significant difference in pain relief between groups; however, adverse events were more frequent in the combination arms. Due to heterogeneity and the relative poor quality of RCTs, the benefit of combination therapy in patients with neuropathic cancer pain cannot be discounted and therefore clinicians should balance potential benefits against the recognised adverse effects of combination therapy. ${ }^{49}$

European Association of Palliative Care guidance recommends that amitriptyline or gabapentin should be considered for patients with neuropathic cancer pain that is only partially responsive to opioid analgesia. ${ }^{15}$ The combination of an opioid with these drugs is more likely to cause adverse central nervous symptoms and so careful dose titration and review should occur.

\section{> Practice point: Gabapentin, pregabalin, amitriptyline or} duloxetine should be considered for patients with neuropathic cancer pain that is only partially responsive to opioid analgesia. Adverse central nervous symptoms are common with coanalgesic drugs and clinicians should balance any potential benefits against recognised adverse effects of combining opioids with coanalgesics.

\section{Conclusion}

There are some recent developments in the pharmacological management of pain in palliative care. There are, however, key gaps in the evidence and further research studies in all drug classes are needed. Patients' treatment must be individualised, weighing up the risks and benefits of medication, while considering patient comorbidities. A reassessment of response (beneficial and adverse effects) to medications must be undertaken frequently and drugs should be stopped if there is no sign of clinical improvement or unmanageable adverse effects. Specialist advice should be sought if unsure.

\section{References}

1 Star A, Boland JW. Updates in palliative care - recent advancements in the pharmacological management of symptoms. Clin Med 2018;18:11-6.

2 Natoli S, Lazzari M, Dauri M. Open questions in the treatment of cancer pain: time for a strong evidence-based approach? Expert Opin Pharmacother 2015;16:1-4.

3 World Health Organization. Cancer pain relief. Geneva: WHO, 1986.

4 Carlson CL. Effectiveness of the World Health Organization cancer pain relief guidelines: an integrative review. J Pain Res 2016;9: 515-34.
5 Wiffen PJ, Derry S, Moore RA et al. Oral paracetamol (acetaminophen) for cancer pain. Cochrane Database Syst Rev 2017;7:CD012637.

6 Derry S, Wiffen PJ, Moore RA et al. Oral nonsteroidal antiinflammatory drugs (NSAIDs) for cancer pain in adults. Cochrane Database Syst Rev 2017;7:CD012638.

7 Bhala N, Emberson J, Merhi A et al. Vascular and upper gastrointestinal effects of non-steroidal anti-inflammatory drugs: metaanalyses of individual participant data from randomised trials. Lancet 2013;382:769-79.

8 Nissen SE, Yeomans ND, Solomon DH et al. Cardiovascular Safety of Celecoxib, Naproxen, or Ibuprofen for Arthritis. N Engl ] Med 2016;375:2519-29.

9 Twycross R, Wilcock A, Howard P. Palliative Care Formulary. 6th edn. Nottingham: Palliativedrugs.com Ltd, 2017.

10 Dickman A. Drugs in Palliative Care. Second Edition. Oxford: OUP, 2012.

11 Scarpignato C, Lanas A, Blandizzi C et al. Safe prescribing of nonsteroidal anti-inflammatory drugs in patients with osteoarthritis an expert consensus addressing benefits as well as gastrointestinal and cardiovascular risks. BMC Med 2015:13:55.

12 National Institute for Heath and Care Excellence. Osteoarthritis: care and management: NICE Guideline (CG177). NICE, 2014. nice.org. uk/guidance/gc177 [Accessed 27 November 2017].

13 Fine $P$. Treatment guidelines for the pharmacological management of pain in older persons. Pain Medicine 2012;13:S57-S66.

14 Crews KR, Caudle KE, Dunnenberger HM et al. Considerations for the utility of the CPIC Guideline for CYP2D6 genotype and codeine therapy. Clin Chem 2015;61:775-6.

15 Caraceni A, Hanks G, Kaasa S et al. Use of opioid analgesics in the treatment of cancer pain: evidence-based recommendations from the EAPC. Lancet Oncol 2012;13:e58-68.

16 National Institute for Health and Care Excellence. Palliative care for adults: strong opioids for pain relief: NICE Guideline (CG140). NICE, 2012. nice.org.uk/guidance/cg140 [Accessed 27 November 2017].

17 Schmidt-Hansen M, Bennett MI, Arnold S et al. Oxycodone for cancer-related pain. Cochrane Database Syst Rev 2017:8:CD003870.

18 Els C, Jackson TD, Kunyk D et al. Adverse events associated with medium and long-term use of opioids for chronic non-cancer pain: an overview of Cochrane Reviews. Cochrane Database Syst Rev 2017:10:CD012509.

19 Corli O, Floriani I, Roberto A et al. Are strong opioids equally effective and safe in the treatment of chronic cancer pain? A multicenter randomized phase IV 'real life' trial on the variability of response to opioids. Annals Oncol 2016;27:1107-15.

20 Davies AN, Dickman A, Reid C, Stevens AM, Zeppetella G. The management of cancer-related breakthrough pain: recommendations of a task group of the Science Committee of the Association for Palliative Medicine of Great Britain and Ireland. Eur J Pain 2009;13:331-8.

21 Boland JW, Boland EG. Pharmacological therapies for opioid induced constipation in adults with cancer. BMJ 2017;358:j3313.

22 Abdulla A, Adams N, Bone M et al. Guidance on the management of pain in older people. Age Ageing 2013:42:1-57.

23 von Gunten CF. The Pendulum Swings for Opioid Prescribing. J Palliat Med 2016;19:348.

24 Boland JW, McWilliams K, Ahmedzai SH, Pockley AG. Effects of opioids on immunologic parameters that are relevant to antitumour immune potential in patients with cancer: a systematic literature review. $\mathrm{Br}$ ] Cancer 2014;111:866-73.

25 Boland JW, Pockley AG. Influence of opioids on immune function in patients with cancer pain: from bench to bedside. $\mathrm{Br}$ ] Pharmacol 2017; doi: 10.1111/bph.13903. [Epub ahead of print].

26 Chang WP, Lin CC. Use of opioid analgesics or sleeping medication and survival of cancer patients. Eur J Oncol Nurs 2015;19:199-206.

27 Boland JW, Ziegler L, Boland EG, McDermid K, Bennett MI. Is regular systemic opioid analgesia associated with shorter survival in adult patients with cancer? A systematic literature review. Pain 2015:156:2152-63. 
28 Boland JW, Allgar V, Boland EG et al. Effect of opioids and benzodiazepines on clinical outcomes in patients receiving palliative care: an exploratory analysis. J Palliat Med 2017;20:1274-9.

29 Roxburgh CS, McMillan DC. Cancer and systemic inflammation: treat the tumour and treat the host. $\mathrm{Br}$ ] Cancer 2014;110:1409-12.

30 Laird BJ, McMillan DC, Fayers P et al. The systemic inflammatory response and its relationship to pain and other symptoms in advanced cancer. Oncologist 2013;18:1050-5.

31 Smith D, Wilkie R, Uthman O, Jordan JL, McBeth J. Chronic pain and mortality: a systematic review. PloS one 2014;9:e99048.

32 Halabi S, Vogelzang NJ, Kornblith AB et al. Pain predicts overall survival in men with metastatic castration-refractory prostate cancer. J Clin Oncol 2008;26:2544-9.

33 Boland J, Boland E, Brooks D. Importance of the correct diagnosis of opioid-induced respiratory depression in adult cancer patients and titration of naloxone. Clin Med 2013;13:149-51.

34 Royal College of Anaesthesia, Faculty of Pain Medicine. Opioids Aware. www.rcoa.ac.uk/faculty-of-pain-medicine/opioids-aware [Accessed 27 November 2017].

35 Velayudhan A, Bellingham G, Morley-Forster P. Opioid-induced hyperalgesia. Cont Ed Anaesth Crit Care Pain 2014;14:125-9.

36 Riley ], Branford R, Droney ] et al. Morphine or oxycodone for cancer-related pain? A randomised control, open-label, controlled trial. J Pain Sympt Manage 2015;49;161-72.

37 Mercadante S, Ferrera P, Villari P et al. Frequency, indications, outcomes and predictive factors of opioid switching in an acute palliative care unit. J Pain Symptom Manage 2009;37:632-41.

38 Drewes AM, Jensen RD, Nielsen LM et al. Differences between opioids: pharmacological, experimental, clinical and economical perspectives. Br J Clin Pharmacol 2013;75:60-78.

39 Palliative Care Adult Network Guidelines Plus. book.pallcare.info [Accessed 11 January 2018].

40 Langford RM, Knaggs R, Farquhar-Smith P, Dickenson AH. Is tapentadol different from classical opioids? A review of the evidence. Br J Pain 2016;10:217-21.
41 Tzschentke TM, Christoph T, Kögel BY. The mu-opioid receptor agonist/noradrenaline reuptake inhibition (MOR-NRI) concept in analgesia: the case of tapentadol. CNS Drugs 2014;28:319-29.

42 Wiffen PJ, Derry S, Naessens K, Bell RF. Oral tapentadol for cancer pain. Cochrane Database Syst Rev 2015:CD011460.

43 Carmona-Bayonas A, Jiménez Fonseca P, Virizuela Echaburu J. Tapentadol for cancer pain management: a narrative review. Pain Pract 2017;17:1075-88

44 Baron R, Eberhart L, Kern KU et al. Tapentadol prolonged release for chronic pain: a review of clinical trials and 5 years of routine clinical practice data. Pain Pract 2017;17:678-700.

45 Smith EM, Pang H, Cirrincione $\mathrm{C}$ et al. Effect of duloxetine on pain, function, and quality of life among patients with chemotherapy-induced painful peripheral neuropathy: a randomized clinical trial. JAMA 2013;309:1359-67.

46 National Institute for Health and Care Excellence. Neuropathic pain in adults: pharmacological management in non-specialist set tings: NICE Guideline (CG 173). NICE, 2013. nice.org.uk/guidance/ cg173 [Accessed 27 November 2017].

47 Mishra S, Bhatnagar S, Goyal GN et al. A comparative efficacy of amitriptyline, gabapentin, and pregabalin in neuropathic cancer pain: a prospective randomized double-blind placebo-controlled study. Am J Hosp Palliat Care 2012;29:177-82.

48 Bennett MI, Laird B, van Litsenburg C, Nimour M. Pregabalin for the management of neuropathic pain in adults with cancer: a systematic review of the literature. Pain Med 2013;14:1681-8.

49 Kane CM, Mulvey MR, Wright S et al. Opioids combined with antidepressants or antiepileptic drugs for cancer pain: systematic review and meta-analysis. Palliat Med 2017:269216317711826.

Address for correspondence: Dr Jason W Boland, Wolfson

Palliative Care Research Centre, Hull York Medical School, Cottingham Rd, Hull HU6 7RX, Yorkshire, UK.

Email: jason.boland@hyms.ac.uk 